\title{
Modeling of Hysteresis and Magnetization Curves for Hexagonally Ordered Electrodeposited Nanowires
}

\author{
Petru S. Fodor \\ Cleveland State University, p.fodor@csuohio.edu \\ Georgy M. Tsoi \\ Wayne State University \\ Lowell E. Wenger \\ Wayne State University, wenger@physics.wayne.edu
}

Follow this and additional works at: https://engagedscholarship.csuohio.edu/sciphysics_facpub

Part of the Physics Commons

How does access to this work benefit you? Let us know!

Publisher's Statement

(C) 2003 American Institute of Physics.

\section{Repository Citation}

Fodor, Petru S.; Tsoi, Georgy M.; and Wenger, Lowell E., "Modeling of Hysteresis and Magnetization Curves for Hexagonally Ordered Electrodeposited Nanowires" (2003). Physics Faculty Publications. 204.

https://engagedscholarship.csuohio.edu/sciphysics_facpub/204

This Article is brought to you for free and open access by the Physics Department at EngagedScholarship@CSU. It has been accepted for inclusion in Physics Faculty Publications by an authorized administrator of

EngagedScholarship@CSU. For more information, please contact library.es@csuohio.edu. 
Modeling of hysteresis and magnetization curves for hexagonally ordered electrodeposited nanowires

P. S. Fodor, G. M. Tsoi, and L. E. Wenger

Citation: 93, 7438 (2003); doi: 10.1063/1.1541643

View online: http://dx.doi.org/10.1063/1.1541643

View Table of Contents: http://aip.scitation.org/toc/jap/93/10

Published by the American Institute of Physics 


\title{
Modeling of hysteresis and magnetization curves for hexagonally ordered electrodeposited nanowires
}

\author{
P. S. Fodor, G. M. Tsoi, and L. E. Wenger ${ }^{\mathrm{a})}$ \\ Department of Physics and Astronomy, Wayne State University, Detroit, Michigan 48201
}

(Presented on 13 November 2002)

\begin{abstract}
A computational model has been developed to investigate how the magnetostatic interactions affect the hysteresis and magnetization curves for hexagonal arrays of magnetic nanowires. The magnetization coupling between nanowires arises from the stray fields produced by the other nanowires composing the array such that the field at each nanowire is the sum of the external field and the interaction field with the other nanowires. Using only two adjustable parameters: the interaction between nearest neighbors and the width of the Gaussian distribution in switching fields centered around the measured coercivity, simulations are compared with the experimentally measured hysteresis and magnetization curves for electrodeposited $\mathrm{Co}_{0.45} \mathrm{Fe}_{0.55}$ alloy nanowires with diameters from 12 to $48 \mathrm{~nm}$. Excellent agreement is found for all nanowire systems except for the largest diameter arrays where deviations from the Gaussian distribution of switching fields need to be considered. (C) 2003 American Institute of Physics. [DOI: 10.1063/1.1541643]
\end{abstract}

Recently, studies of the magnetic properties of electrodeposited magnetic nanowires in anodic alumina ${ }^{1-3}$ and "track-etch", 4,5 porous membranes have attracted interest due to their prospective application for high density recording media. The porous template route constitutes a promising alternative for the fabrication of magnetic nanostructures due to its capabilities for large area and high aspect ratio nanopatterning. Ferromagnetic transition metals can be easily electrodeposited into these pores to form magnetic nanowires with large uniaxial anisotropy as a result of their high aspect ratio.

Most studies on magnetic nanowire arrays produced in porous templates are concerned with understanding the mechanism for the magnetization reversal in individual nanowires and how their dimensions and microstructure affect their magnetic properties. However, little research has been conducted regarding the effect of the magnetostatic interactions between the nanowires on the magnetic properties of the arrays. Previous computational studies of the long range magnetostatic coupling in nanowire arrays have obtained only qualitative agreement with the experiments ${ }^{5,6}$ or used systems consisting of a small number of nanowires in order to reduce the computational complexity of the problem $^{7}$ so that no attempt could be made to compare the simulations to the experimental data.

In this article a simple computational model will be introduced that reproduces the experimental hysteresis and magnetization curves along the easy magnetization axis for hexagonally ordered $\mathrm{Co}_{0.45} \mathrm{Fe}_{0.55}$ alloy nanowire arrays. For details regarding the preparation of the anodic alumina templates and the fabrication of the nanowires the reader is referred to Refs. 3 and 8. Briefly, ac electrodeposition in highly ordered alumina templates prepared in sulfuric and oxalic acid solutions is used to fabricate arrays of magnetic nano-

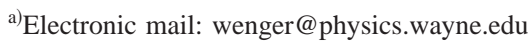

wires of $12 \mu \mathrm{m}$ length and diameter $D_{p}$ ranging from 12 to $48 \mathrm{~nm}$. The pore-to-pore distance $D_{i}$ is 55 and $95 \mathrm{~nm}$ for the templates prepared in sulfuric acid and oxalic acid, respectively. Magnetic measurements performed in a SQUID magnetometer show that the easy magnetization axis is along the nanowires' axis for all the samples studied. This is consistent with the high uniaxial anisotropy along the length of the nanowires arising from the large shape anisotropy and small magnetocrystalline anisotropy measured in $\mathrm{Co}_{0.45} \mathrm{Fe}_{0.55}$ alloys. ${ }^{9}$ The high degree of ordering and well-defined nanowires diameters and spacing makes the magnetic nanowire arrays particularly suitable for comparisons to rigorous computational studies.

Due to the strong shape anisotropy, the individual nanowires are considered to have square hysteresis loops with only two stable magnetic states characterized by a single magnetic domain extending through the entire nanowire for fields applied along the easy magnetization axis. Each nanowire will be treated as a single magnetic moment and coupled to the stray magnetic fields from the other nanowires composing the array. Thus the field at the site $(i, j)$ of a nanowire will be a sum of the applied magnetic field $H$ and the interaction fields from the other nanowires composing the array:

$$
H_{i, j}=H+M \sum_{\substack{l \neq i \\ m \neq j}} \sigma_{l, m} K_{l m, i j},
$$

where $K_{l m, i j}$ is a geometrical coupling factor, $M$ is the magnetic moment of a nanowire, and $\sigma_{l, m}$ equals 1 and -1 depending on the magnetization orientation along the easy magnetization axis being parallel or antiparallel to the field.

The geometrical coupling factor $K_{l m, i j}$ depends in principle on the distance between the interacting nanowires. A simple analogy to the interaction between two parallel point dipoles side-by-side leads to the conclusion that the coupling 

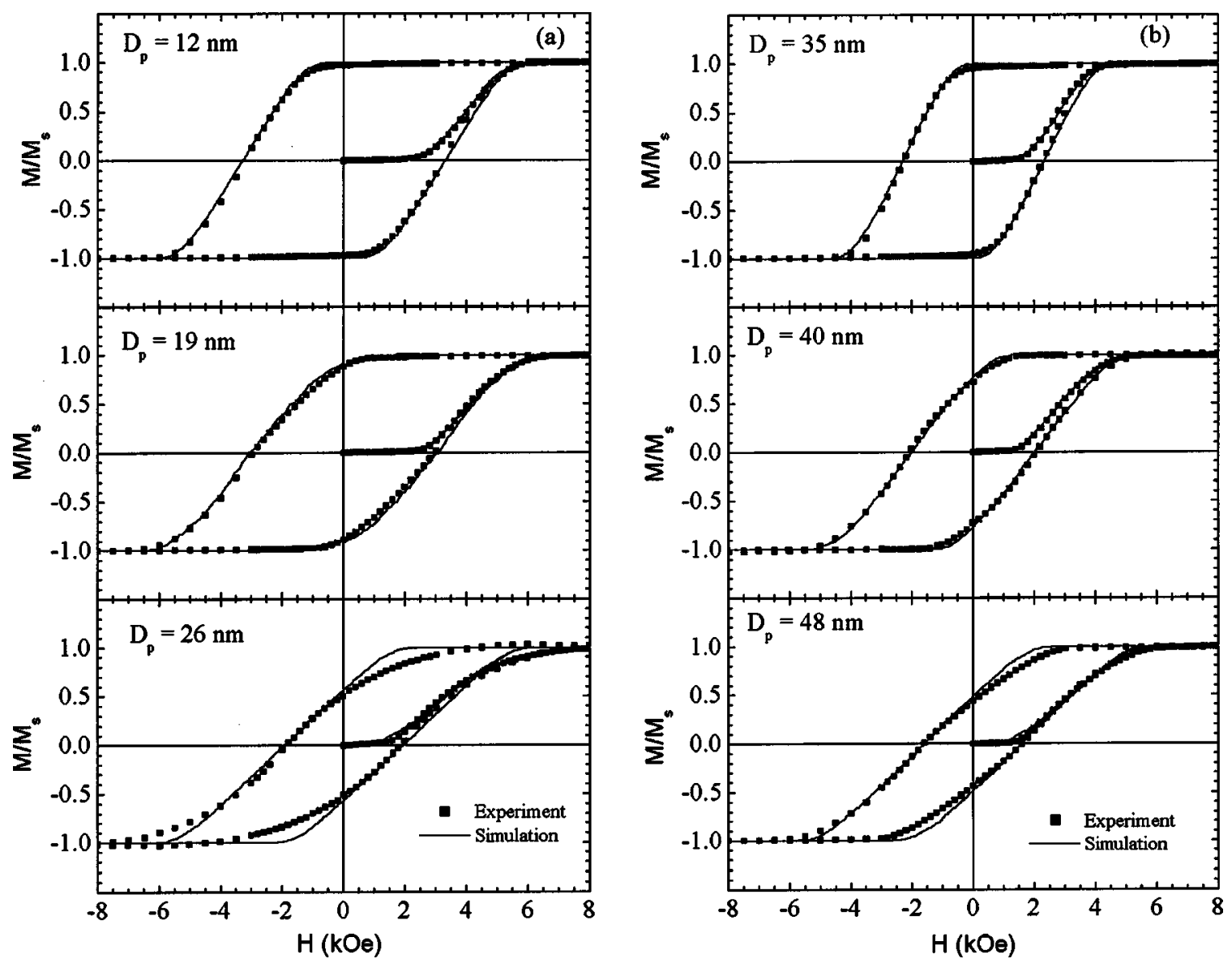

FIG. 1. Hysteresis and magnetization curves along the easy axis for samples prepared in templates with (a) $D_{i}=55 \mathrm{~nm}$ and (b) $D_{i}=95 \mathrm{~nm}$. The lines are best fits of the computer simulations to the data.

factor is inversely proportional to the cube of the distance between the nanowires:

$$
K_{l m, i j}=\frac{K}{D_{i}^{3} \cdot\left[(l-i)^{2}+(m-j)^{2}+(l-i)(m-j)\right]^{3 / 2}},
$$

where $D_{i}$ is the distance between the nearest neighbors. ${ }^{10}$ The factor $K$, which equals 1 for point dipoles, is introduced to account for the fact that the nanowires are not point dipoles. The magnetic field at any nanowire site $(i, j)$ can be then written in the form:

$$
\begin{aligned}
H_{i, j}= & H+H_{d} \\
& \times \sum_{\substack{l \neq i \\
m \neq j}} \sigma_{l, m} \frac{1}{\left[(l-i)^{2}+(m-j)^{2}+(l-i)(m-j)\right]^{3 / 2}},
\end{aligned}
$$

where $H_{d}=K /\left(M \cdot D_{i}^{3}\right)$ is the interaction field between the nearest neighbors and is one of the adjustable parameters in the simulations. Thus the effect of the magnetostatic interactions will be to increase the field experienced by each nanowire above the value of the applied field in a saturated state and to decrease the field at which a nanowire will reverse its magnetization. However, once the magnetization switching takes place, the stray field of the magnetic nanowire will oppose the reversal of the magnetization in the other nano- wires and thus will increase the field at which their magnetization will be reversed. It is very important to keep in mind that the switching field of the individual nanowires is a welldefined characteristic determined by their internal magnetic properties. The magnetostatic interactions only introduce an additional term in the magnetic field, which changes the effective field perceived by a nanowire and not its coercivity. Thus the effect of the magnetostatic interactions on the hysteresis loop of a large array will be to increase the saturation magnetic field and decrease the remanence.

Typically, a $40 \times 40$ two-dimensional hexagonal array with open boundary conditions ${ }^{11}$ is used for most of our simulations, since arrays as large as $100 \times 100$ are found to give similar results while reducing the effect of the boundary conditions. The coercivity of the nanowires forming the arrays is assumed to have a Gaussian distribution with a standard deviation $d H_{c}$ around the experimentally measured coercivity $H_{c}$ for the entire array. To construct the hysteresis and magnetization loops the external field is changed in 50 Oe steps and the effective field at the position of each nanowire is calculated for the new external magnetic field. If the effective field is larger than the nucleation field of the particular nanowire, the magnetization of the nanowire is reversed and the magnetostatic interactions are updated. The iteration continues until none of the nanowires changes its magnetization. A new field is then set and the procedure 
TABLE I. Parameters used in the computer simulations of the hysteresis and magnetization curves.

\begin{tabular}{cccccc}
\hline \hline & $\begin{array}{c}D_{i} \\
(\mathrm{~nm})\end{array}$ & $\begin{array}{c}D_{p} \\
(\mathrm{~nm})\end{array}$ & $\begin{array}{c}H_{c} \\
(\mathrm{Oe})\end{array}$ & $\begin{array}{c}d H_{c} \\
(\mathrm{Oe})\end{array}$ & $\begin{array}{c}H_{d} \\
(\mathrm{Oe})\end{array}$ \\
\hline 1 & 55 & 12 & 3500 & 500 & 125 \\
2 & 55 & 15 & 3400 & 550 & 150 \\
3 & 55 & 19 & 3160 & 600 & 170 \\
4 & 55 & 26 & 2000 & 550 & 240 \\
5 & 55 & 35 & 1740 & 600 & 365 \\
6 & 95 & 35 & 2330 & 500 & 90 \\
7 & 95 & 40 & 2080 & 625 & 150 \\
8 & 95 & 46 & 1780 & 600 & 210 \\
9 & 95 & 48 & 1700 & 600 & 230 \\
\hline \hline
\end{tabular}

repeated. The calculated curves are then compared with the experimental results and the simulation parameters $H_{d}$ and $d H_{c}$ are subsequently modified in order to obtain the best agreements. A similar procedure was previously successful in simulating data from square arrays of single domain particles prepared by interference lithography. ${ }^{12,13}$

Figure 1 displays a comparison between the simulated curves and the experimental data. Taking into account the simplicity of the model used, the agreement of the simulation to the experimental data for both sets of templates is surprisingly good for samples with smaller diameters. For the samples with larger diameters, the agreement between the simulation and the experimental data is poorer as only qualitative agreement is observed. It is believed that these larger diameter samples have a more complex distribution of switching fields and the Gaussian distribution does not truly reflect the characteristics of the system. At the same time, it is very probable for larger diameter samples that there is a spread in the interaction fields as well.

As summarized in Table $\mathrm{I}$, the deviation $d H_{c}$ in the switching field ranges from 500 to 625 Oe. This spread is probably due to compositional differences, crystalline defects, and slight differences in the shape of the nanowires, which affects the local anisotropy. Although no clear diameter dependence is observed for the deviation $d H_{c}$, the interaction parameter $H_{d}$ increases quadratically with the nanowire diameter for both templates as shown in Fig. 2. This quadratic dependence is consistent with an interaction field arising from the surface pole density $\left(\propto M_{s}\left(D_{p}\right)^{2}\right)$ for a uniformly magnetized cylinder. However, the $\left.1 / D_{i}\right)^{3}$ dependence expected for a dipole interaction field as a function of the nearest neighbor distance is not observed. This may result from the idealized treatment of a nanowire being a single magnetic dipole with magnetic charges at its ends. Recent magnetic force microscopy studies ${ }^{4}$ have shown that magnetic nanowires can sustain magnetic domains, which implies that the magnetic charges that give rise to the magnetostatic interactions between the nanowires can be distributed along the nanowire length, making a theoretical study of the interaction strength a much more difficult task.

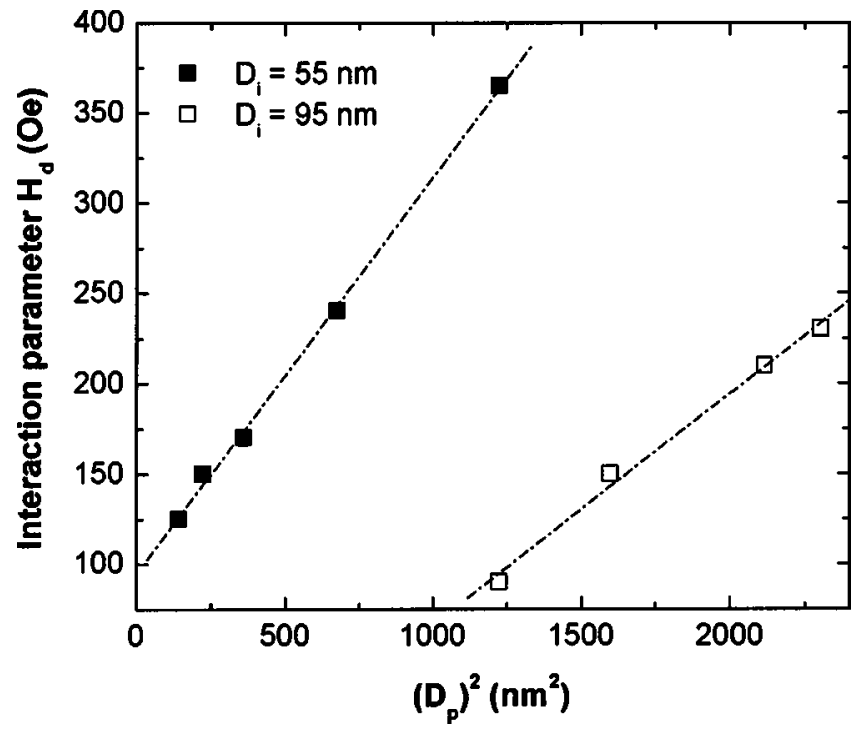

FIG. 2. Dependence of the interaction parameter $H_{d}$ on the nanowire diameter $D_{p}$ for templates with $D_{i}=55$ and $95 \mathrm{~nm}$, respectively.

In summary, simulations on magnetostatically interacting nanowire arrays using a simplified model that considers dipole-dipole-like interactions have been performed. The remanence ratio is found to decrease, while the saturation field increases with increasing interaction strength. The reduction of the magnetostatic interactions in these nanowire systems is critical in order to satisfy the technological requirement that the nanowire switching be independent of the state of its neighbors.

This work is supported in part by the National Science Foundation through Grant No. DGE-9870720.

${ }^{1}$ D. J. Sellmyer, M. Zheng, and R. Skomski, J. Phys.: Condens. Matter 13, R433 (2001).

${ }^{2}$ R. M. Metzger, V. V. Konovalov, M. Sun, T. Xu, G. Zangari, B. Xu, M. Benakli, and W. D. Doyle, IEEE Trans. Magn. 36, 30 (2000).

${ }^{3}$ P. S. Fodor, G. M. Tsoi, and L. E. Wenger, J. Appl. Phys. 91, 8879 (2002).

${ }^{4}$ Y. Henry, K. Ounadjela, L. Piraux, S. Dubois, J. M. George, and J. L. Duvail, Eur. Phys. J. B 20, 35 (2001).

${ }^{5}$ V. Raposos, J. M. Garcia, J. M. González, and M. Vázquez, J. Magn. Magn. Mater. 222, 227 (2000).

${ }^{6}$ J. M. Garcia, A. Asenjo, J. Velásquez, D. Garcia, M. Vázquez, P. Aranda, and E. Ruiz-Hitzky, J. Appl. Phys. 85, 5480 (1999).

${ }^{7}$ R. Hertel, J. Appl. Phys. 90, 5752 (2001).

${ }^{8}$ P. S. Fodor, G. M. Tsoi, and L. E. Wenger, Phys. Rev. B (submitted).

${ }^{9}$ C. Kittel, Rev. Mod. Phys. 21, 541 (1949).

${ }^{10}$ The assignment of the nanowires positions $(i, j)$ are based on a hexagonal array with its principal axes being at a $60^{\circ}$ angle with respect to each other.

${ }^{11}$ Since the $40 \times 40$ array is comparable to the average ordered domain size of the hexagonal arrangement $(\sim 2 \mu \mathrm{m})$, the open boundary conditions probably better represent the actual nanowire arrays than periodic boundary conditions as the interactions across the domain boundaries are not easily quantifiable using either approach.

${ }^{12}$ C. A. Ross, M. Rarhoud, M. Hwang, H. I. Smith, M. Redjdal, and F. B. Humphrey, J. Appl. Phys. 89, 1310 (2001).

${ }^{13}$ M. Hwang, M. Farhoud, Y. Hao, M. Walsh, T. A. Savas, H. I. Smith, and C. A. Ross, IEEE Trans. Magn. 36, 3173 (2000). 